\title{
Brain malformation-congenital heart disease-postaxial polydactyly syndrome
}

INSERM

\section{Source}

INSERM. (1999). Orphanet: an online rare disease and orphan drug data base. Brain malformation-congenital heart disease-postaxial polydactyly syndrome. ORPHA:75389

Goossens-Devriendt syndrome is characterised by intrauterine growth retardation, a congenital heart defect, postaxial polydactyly, a brain malformation, abnormal hair with temporal balding, and marked facial dysmorphism. It has been reported in two siblings from unrelated parents. One of the siblings died and the surviving patient showed postnatal growth retardation and severe developmental delay. 